\title{
Desenvolvimento do processo de enfermagem conforme necessidades psicobiológicas autorreferidas por estudantes
}

\author{
Development of the nursing process according to psychobiological needs self-referred by students \\ Desarrollo del Proceso de Enfermería de acuerdo con las necesidades psicobiológicas autoinformadas por \\ los estudiantes
}

\author{
Diéssica Roggia Piexak' ๑, Munique Pimentel Gomes ${ }^{\circledR} \odot$, Cintia Camila Santos de Souza Costa ${ }^{\circledR}$,

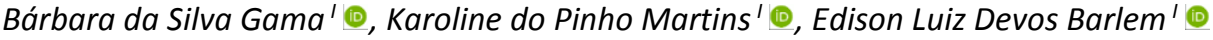

'Universidade Federal do Rio Grande, Rio Grande, RS, Brasil

\begin{abstract}
RESUMO
Objetivo: identificar os diagnósticos de enfermagem prevalentes em estudantes de enfermagem, na perspectiva das necessidades psicobiológicas autorreferidas, descrever os resultados esperados e propor intervenções de enfermagem. Método: estudo quantitativo, exploratório e descritivo, realizado em 2018 com 156 estudantes do curso de graduação em enfermagem, após aprovação do Comitê de Ética em Pesquisa. Utilizou-se um questionário autoaplicável e a análise foi a partir do software estatístico SPSS, por meio de estatística descritiva. Resultados: os diagnósticos de enfermagem mais prevalentes foram: fadiga $(93,6 \%)$, insônia $(67,5 \%)$, estilo de vida sedentário $(54,5 \%)$ e constipação $(53,8 \%)$. Foram elencados objetivos a serem alcançados a partir desses diagnósticos, e então determinadas possíveis intervenções. Conclusão: observou-se que, dos quatro diagnósticos mais prevalentes, dois apresentavam o estresse e a ansiedade como fatores contribuintes para o surgimento. Assim, faz-se necessário que as instituições de ensino superior adotem medidas de enfrentamento e promoção da saúde dos estudantes frente a esses aspectos.
\end{abstract}

Descritores: Enfermagem; Estudantes de Enfermagem; Processo de Enfermagem; Diagnóstico de Enfermagem.

\begin{abstract}
Objective: to identify the nursing diagnoses prevalent in nursing students, from self-reported psychobiological needs, to describe the results expected, and propose nursing interventions. Method: this quantitative, exploratory, descriptive study of 156 undergraduate nursing students was conducted in 2018, after approval by the research ethics committee. A selfadministered questionnaire was used and data analysis was based on the statistical software SPSS, by means of descriptive statistics. Results: the most prevalent nursing diagnoses were: fatigue (93.6\%), insomnia (67.5\%), sedentary lifestyle (54.5\%) and constipation (53.8\%). Objectives to be achieved from these diagnoses were listed, and then possible interventions were determined. Conclusion: of the four most prevalent diagnoses, two involved stress and anxiety as contributing factors. Higher education institutions should thus adopt measures to address these conditions and promote students' health accordingly. Descriptors: Nursing; Student, Nursing; Nursing Process; Nursing Diagnosis.
\end{abstract}

\section{RESUMEN}

Objetivo: identificar los diagnósticos de enfermería prevalentes en estudiantes de enfermería, a partir de las necesidades psicobiológicas autoinformadas, describir los resultados esperados y proponer intervenciones de enfermería. Método: este estudio cuantitativo, exploratorio y descriptivo de 156 estudiantes de pregrado en enfermería se realizó en 2018, luego de la aprobación del comité de ética en investigación. Se utilizó un cuestionario autoadministrado y el análisis de los datos se basó en el software estadístico SPSS, mediante estadística descriptiva. Resultados: los diagnósticos de enfermería más prevalentes fueron: fatiga $(93,6 \%)$, insomnio $(67,5 \%)$, sedentarismo $(54,5 \%)$ y estreñimiento $(53,8 \%)$. Se enumeraron los objetivos a alcanzar a partir de estos diagnósticos y luego se determinaron las posibles intervenciones. Conclusión: de los cuatro diagnósticos más prevalentes, dos involucraron estrés y ansiedad como factores contribuyentes. Por tanto, las instituciones de educación superior deberían adoptar medidas para abordar estas condiciones y promover la salud de los estudiantes en consecuencia. Descriptores: Enfermería; Estudiantes de Enfermería; Proceso de Enfermería; Diagnóstico de Enfermería.

\section{INTRODUÇÃO}

Ao ingressar na universidade, os estudantes de graduação em enfermagem gradativamente assumem maiores responsabilidades e adquirem mais autonomia. A rotina acadêmica traz consigo diversas mudanças nos hábitos cotidianos, pois muitas são as cobranças acadêmicas até então desconhecidas para a maioria desses estudantes. Manter um rendimento acadêmico satisfatório, muitas vezes, desencadeia ansiedade ou outros problemas relacionados a saúde mental e muitos estudantes acabam optando por hábitos de vida menos saudáveis, onde a médio ou a longo prazo, podem causar deterioração da própria saúde, gerando mal-estar e até mesmo adoecimento ${ }^{1,2}$. 
Pesquisas demonstram dados preocupantes frente às Necessidades Humanas Básicas (NHBs) de estudantes universitários, especialmente as psicobiológicas. Essas vão desde alimentação baseada em ingestão excessiva de carboidratos, gorduras e doces, alta taxa de sedentarismo e consumo de bebidas alcoólicas ${ }^{3}$; uso de analgésicos, tranquilizantes ou sedativos no intuito de reduzir a ansiedade e melhora da qualidade do sono ${ }^{4}$; automedicação devido a cefaleia, dores abdominais, febre, resfriado e distúrbios gastrointestinais ${ }^{5}$; são considerados, ainda, fisicamente inativos ou insuficientemente ativos ${ }^{6}$ e apresentam má qualidade do sono ${ }^{7}$. Destaca-se, também, que o sedentarismo corrobora para o sobrepeso e está entre as principais causas de desenvolvimento de diabetes, hipertensão arterial, dislipidemias e outras doenças crônicas ${ }^{1}$.

Alterações no sono, juntamente com desgaste físico e mental, desânimo, estresse, cefaleias e dores musculares, são manifestações comumente encontradas em estudantes de graduação em enfermagem, e podem estar relacionadas com sintomas de exaustão emocional ${ }^{8}$.

Nesse sentido, desenvolver o Processo de Enfermagem na perspectiva das necessidades psicobiológicas autorreferidas por estudantes de enfermagem torna-se imprescindível para a elaboração de estratégias efetivas de promoção da saúde, prevenção e recuperação de doenças em um ambiente em que se deveria primar pelo cuidado do futuro cuidador, estimulando principalmente o autocuidado. Pois a melhoria da qualidade de vida do estudante de graduação em enfermagem tem influência direta no processo de humanização do cuidado prestado por este ${ }^{9}$.

Portanto, este estudo teve por objetivos identificar os diagnósticos de enfermagem prevalentes em estudantes de enfermagem, na perspectiva das necessidades psicobiológicas autorreferidas, descrever os resultados esperados e propor intervenções de enfermagem.

\section{REVISÃO DA LITERATURA}

Adotaram-se os referenciais teóricos: $\mathrm{NHBs}^{10}$, a Taxonomia Diagnóstica da North American Nursing Diagnosis Association (NANDA International) ${ }^{11}$, a Classificação das Intervenções de Enfermagem (NIC) ${ }^{12}$ e a Classificação de Resultados Esperados (NOC) $)^{13}$ para o desenvolvimento das cinco etapas do Processo de Enfermagem.

O Processo de Enfermagem está regulamentado através da Resolução $n^{\circ}$ 358/09. Essa resolução define cinco etapas, inter-relacionadas e interdependentes, para a aplicação do Processo, sendo essas: realização do histórico de enfermagem; definição de diagnóstico de enfermagem; planejamento de intervenções; implementação de intervenções; e avaliação das intervenções realizadas ${ }^{14}$.

A execução de todas essas etapas proporciona que o enfermeiro desempenhe suas atividades de maneira sistemática, mas sem desconsiderar as particularidades de cada indivíduo, e assim organizar as condições necessárias para ofertar um cuidado de qualidade, possibilitando o registro de sua prática durante todo processo ${ }^{15}$. Exige do profissional conhecimento científico, habilidades técnicas e atitudes baseadas na eticidade e na responsabilidade com o paciente assistido, permitindo ainda, o controle organizacional com relação aos recursos físicos e materiais incluídos no processo ${ }^{16}$. Além disso, a linguagem padronizada, por meio do uso da teoria das NHBs e da NANDA-I, NIC e NOC, facilitam o ensino e a tomada de decisões, favorecendo a pesquisa e, consequentemente, o fortalecimento da ciência de enfermagem ${ }^{17}$.

Ademais, percebe-se que há escassez na literatura científica sobre a investigação das NHBs psicobiológicas de estudantes de graduação em enfermagem por meio da ferramenta científica do Processo de Enfermagem articulado à NANDA-I, NIC e NOC, constituindo-se como uma importante fonte de informações para o cuidado desses futuros profissionais.

\section{MÉTODO}

Estudo quantitativo, exploratório e descritivo, realizado em uma instituição de ensino superior do Sul do Brasil, entre os meses de março e abril de 2018. A matriz didático-pedagógica do curso conta com 10 semestres, nos turnos da manhã e tarde, com ingresso semestral de 30 estudantes, por meio do Sistema de Seleção Unificada (SISU), tendo como carga horária total 4.140 horas, distribuídas entre disciplinas teóricas e teórico-práticas obrigatórias, incluindo o Projeto e o Trabalho de Conclusão de Curso, estágios supervisionados e atividades complementares, cujo objetivo é a formação de um enfermeiro generalista inserido no contexto socioambiental ${ }^{18}$

A amostra do estudo foi constituída por 156 estudantes do curso de graduação em enfermagem, dos 10 semestres, que aceitaram participar. Estes foram selecionados mediante amostragem não probabilística por conveniência, conforme sua presença nos locais de estudo e disponibilidade para participar da pesquisa ${ }^{19}$

Para garantir a confiabilidade dos dados, uma fórmula específica foi utilizada para determinar o mínimo de participantes necessários ao estudo. A seleção do tamanho amostral seguiu o critério proposto por Hill e Hill ${ }^{20}$. Esta regra oportuniza estimar o mínimo tamanho amostral para que seja possível a realização de determinados procedimentos estatísticos, uma vez que procedimentos diferentes apresentam necessidades específicas de número de 
participantes. Conhecendo previamente o total da população, composta por 260 estudantes, e aplicando-se a fórmula, chegou-se ao número mínimo de 127 participantes.

Os critérios de inclusão para seleção dos participantes compreenderam: ser estudante do curso de graduação em enfermagem da instituição e estar presente em sala de aula no período de coleta dos dados. Os critérios de exclusão estavam limitados a: estarem em atestado médico ou em situação de trancamento do curso.

A coleta foi efetuada de forma coletiva, por meio da disponibilização do questionário estruturado e autoaplicável em papel pardo e sem identificação, em sala de aula, onde foi realizado o convite para participação, apresentando e descrevendo resumidamente os objetivos da pesquisa. Após a aceitação, houve a entrega dos questionários. Ao término do preenchimento foram recolhidos pela pesquisadora responsável. Dos estudantes presentes no momento da coleta, apenas sete recusaram-se a participar.

Como instrumento de coleta de dados, elaborou-se um questionário estruturado e autoaplicável, com perguntas abertas e fechadas. Ele foi desenvolvido com base na literatura científica sobre as principais NHBs identificadas em estudantes de graduação e posteriormente relacionadas com os possíveis Diagnósticos de Enfermagem da NANDA International $2015-2017^{11}$. O questionário continha variáveis para a caracterização dos participantes, como sexo, idade, semestre atual, entre outras, e questões relacionadas aos diagnósticos da NANDA onde, por meio de questões de múltipla escolha, perguntava-se quais eram as características (sinais e sintomas) que o estudante apresentava e se ele identificava que essas ocorriam devido a alguns dos fatores relacionados apresentados.

Destaca-se que, antes do início da coleta dos dados, foi realizado um teste do instrumento, aplicando-o a 10 estudantes de graduação em enfermagem da instituição, selecionados conforme a sua disposição para responder ao instrumento. Considerando o preenchimento por pelo menos um representante de cada semestre do curso, composto por 10, os quais não fizeram parte da amostra mínima, com o intuito de readequar e melhorar o instrumento.

A análise dos dados foi realizada a partir do software estatístico Statistical Package for Social Sciences (SPSS) versão 20.0, por meio de estatística descritiva. O desenvolvimento do processo do raciocínio do diagnóstico de enfermagem se deu por meio de duas etapas, conforme Risner ${ }^{21}$. Na primeira fase, através de um processo de análise e síntese dos dados coletados, faz-se um julgamento clínico das respostas do indivíduo aos processos vitais. Na segunda fase denomina-se o diagnóstico de enfermagem propriamente dito pela taxonomia da North American Nursing Diagnosis Association $^{11}$. Após esta análise, foram propostos pelas próprias pesquisadoras do estudo as intervenções de enfermagem e os resultados esperados, conforme as taxonomias Nursing Interventions Classification (NIC) ${ }^{11}$ e Nursing Outcomes Classification (NOC) ${ }^{13}$, respectivamente.

O estudo respeitou os preceitos éticos da Resolução no 466, de 12 de dezembro de 2012, do Conselho Nacional de Saúde, e foi aprovado pelo Comitê de Ética da instituição (CAAE no 81442517.8.0000.5324).

\section{RESULTADOS}

Entre os 156 participantes, a maioria dos estudantes de graduação em enfermagem era do sexo feminino (90,4\%, $\mathrm{n}=141)$, com média de 23,9 anos de idade, e estava cursando o primeiro ano da faculdade $(33,4 \%, \mathrm{n}=52)$. Ao serem questionados se praticavam atividade física, $42,7 \%(n=67)$ afirmaram não praticar, e, dentre os que praticavam, as atividades mais citadas foram: academia de musculação; caminhadas e ciclismo, com frequência média de três vezes na semana. O Índice de Massa Corporal (IMC) de cada participante foi calculado e está descrito na Tabela 1 abaixo, com base nos parâmetros disponibilizados pela Organização Mundial da Saúde (OMS) dos graus/classes de obesidade ${ }^{22}$.

TABELA 1: Classificação de obesidade conforme IMC. Rio Grande, RS, Brasil, 2018.

\begin{tabular}{|c|c|c|c|}
\hline IMC $\left(\mathrm{kg} / \mathrm{m}^{2}\right)$ & Classificação & Obesidade Grau/Classe & Frequência (\%) \\
\hline$<18,5$ & Magro ou baixo peso & 0 & $3,9 \%(n=6)$ \\
\hline $18,5-24,9$ & Normal ou eutrófico & 0 & $59,6 \%(n=90)$ \\
\hline $25-29,9$ & Sobrepeso ou pré-obeso & 0 & $23,2 \%(n=35)$ \\
\hline $30-34,9$ & Obesidade & 1 & $7,2 \%(n=11)$ \\
\hline $35-39,9$ & Obesidade & II & $4,6 \%(n=7)$ \\
\hline$\geq 40$ & Obesidade Grave & III & $1,4 \%(n=2)$ \\
\hline
\end{tabular}

Fonte: próprio autor (2018).

Em relação ao uso de drogas lícitas, $47,1 \%(n=73)$ dos estudantes responderam que utilizavam, dentre as drogas citadas estavam: cigarro $(5,2 \%, n=8)$, com média de 10,6 cigarros/dia; bebida alcoólica $(32,9 \%, n=51)$, destacando-se principalmente a cerveja e vinho, ingerindo entre um e dois litros, de três a quatro vezes ao mês; medicamentos controlados (12,3\%, n=19), em que os principais apontados foram antidepressivos e ansiolíticos. $E$, no que diz respeito às drogas ilícitas, 94,8\% ( $n=145$ ) dos estudantes afirmaram não utilizar, enquanto $2,6 \%(n=4)$ não quiseram responder e os demais deixaram a questão em branco. 
Quando questionados se possuíam algum diagnóstico médico prévio, $21,3 \%$ ( $n=33$ ) responderam que sim, sendo citados principalmente: depressão; ansiedade; síndrome do pânico; asma; hipo/hipertireoidismo; enxaqueca; e síndrome do intestino irritável. Disseram fazer uso de medicação contínua $54,5 \%(n=85)$, sendo estes, além dos já descritos no parágrafo anterior, medicamentos para o tratamento de asma crônica, anti-inflamatórios não esteroidais, suplementos vitamínicos e anticoncepcionais orais.

A Figura 1 apresenta os diagnósticos de enfermagem mais prevalentes nos estudantes de graduação em enfermagem a partir da autorreferência, com identificação das características definidoras apresentadas e dos fatores relacionados.

\begin{tabular}{|c|c|c|}
\hline Diagnósticos de Enfermagem & Características definidoras & Fatores relacionados \\
\hline $\begin{array}{l}\text { Fadiga } \\
93,6 \% \text { ( } n=146 \text { ) } \\
\text { Sensação opressiva e } \\
\text { prolongada de exaustão e } \\
\text { capacidade diminuída para } \\
\text { realizar trabalho físico e } \\
\text { mental no nível habitual. }\end{array}$ & $\begin{array}{l}\text { Alteração na concentração: 50,6\% ( } n=79 \text { ) } \\
\text { Alteração na libido: } 13,5 \% \text { ( } n=21 \text { ) } \\
\text { Aumento da necessidade de descanso: 64,7\% } \\
\text { ( } n=101 \text { ) } \\
\text { Aumento dos sintomas físicos: } \\
\text { 19,2\% ( } n=30 \text { ) } \\
\text { Cansaço: } 76,3 \% \text { ( } n=119 \text { ) } \\
\text { Capacidade prejudicada para manter as } \\
\text { rotinas habituais: } 19,9 \% \text { ( } n=31 \text { ) } \\
\text { Culpa devida à dificuldade para cumprir com } \\
\text { minhas responsabilidades: } 39,1 \% \text { ( } n=61 \text { ) } \\
\text { Desempenho ineficaz de papéis: } 9,6 \% \text { ( } n=15 \text { ) } \\
\text { Desinteresse quanto ao ambiente que me } \\
\text { cerca: } 14,1 \% \text { ( } n=22 \text { ) } \\
\text { Energia insuficiente: } 39,7 \% \text { ( } n=62 \text { ) } \\
\text { Introspecção: } 14,1 \% \text { ( } n=22 \text { ) } \\
\text { Padrão de sono não restaurador: } 19,9 \% \text { ( } n=31 \text { ) } \\
\text { Sonolência: } 50 \% \text { ( } n=78 \text { ) }\end{array}$ & $\begin{array}{l}\text { Ansiedade: } 52,6 \% \text { ( } n=82 \text { ) } \\
\text { Depressão: } 9 \% \text { ( } n=14) \\
\text { Estilo de vida não estimulante: } 12,2 \% \text { ( } n=19) \\
\text { Estressores: } 52,6 \% \text { ( } n=82) \\
\text { Evento de vida negativo: } 9,6 \%(n=15) \\
\text { Exigências do trabalho: } 36,5 \%(n=57) \\
\text { Privação do sono: } 31,4 \%(n=49) \\
\text { OUTROS MOTIVOS: } \\
\text { Atividades acadêmicas, falta de tempo, } \\
\text { trabalho noturno e estudos, organização do } \\
\text { tempo: } \\
10,9 \%(n=17)\end{array}$ \\
\hline $\begin{array}{l}\text { Insônia } \\
67,5 \% \text { ( } n=104) \\
\text { Distúrbio na quantidade e } \\
\text { qualidade do sono que } \\
\text { prejudica o funcionamento }\end{array}$ & $\begin{array}{l}\text { Alteração na concentração: } 50,6 \% \text { (n=79) } \\
\text { Energia insuficiente: } 39,7 \% \text { ( } n=62) \\
\text { Padrão de sono não restaurador: } 19,9 \% \\
(n=31)\end{array}$ & $\begin{array}{l}\text { Agente farmacológico: } 1,3 \%(n=2) \\
\text { Alterações hormonais: } 4,5 \%(n=7) \\
\text { Ansiedade: } 42,2 \%(n=65) \\
\text { Cochilos frequentes durante o dia: } 6,5 \% \\
(n=10) \\
\text { Depressão: } 7,1 \%(n=11) \\
\text { Desconforto físico: } 6,5 \%(n=10) \\
\text { Estressores: } 39,6 \%(n=61) \\
\text { Medo: } 7,1 \%(n=11) \\
\text { OUTROS MOTIVOS: } \\
\text { Falta de tempo, rotina acadêmica, jornada de } \\
\text { trabalho noturna: } \\
24,7 \%(n=38)\end{array}$ \\
\hline $\begin{array}{l}\text { Estilo de vida sedentário } \\
54,5 \% \text { ( } n=86 \text { ) } \\
\text { Refere-se a um hábito de vida } \\
\text { que se caracteriza por um } \\
\text { baixo nível de atividade física. }\end{array}$ & $\begin{array}{l}\text { Falta de condicionamento físico: } 39,4 \% \\
\text { ( } n=61 \text { ) } \\
\text { Preferência por atividades com pouco } \\
\text { exercício físico: } 20,6 \%(n=32)\end{array}$ & $\begin{array}{l}\text { Interesse insuficiente pela atividade física: } \\
18,7 \%(n=29) \\
\text { Motivação insuficiente para a atividade física: } \\
29 \%(n=45) \\
\text { OUTROS MOTIVOS: } \\
\text { Falta de tempo, falta de dinheiro: } 21,3 \%(n=33)\end{array}$ \\
\hline $\begin{array}{l}\text { Constipação } \\
53,8 \% \text { ( }=83 \text { ) } \\
\text { Diminuição na frequência } \\
\text { normal de evacuação, } \\
\text { acompanhada por eliminação } \\
\text { difícil ou incompleta de fezes } \\
\text { e/ou eliminação de fezes } \\
\text { excessivamente duras e secas }\end{array}$ & $\begin{array}{l}\text { Dor à evacuação: } 3,8 \%(n=6) \\
\text { Dor abdominal: } 14,1 \%(n=22) \\
\text { Esforço para evacuar: } 16,7 \%(n=26) \\
\text { Fezes duras e formadas: } 21,2 \%(n=33) \\
\text { Fezes líquidas: } 4,5 \% \text { ( } n=7) \\
\text { Flatulência (liberação de gases) grave: } 7,1 \% \\
\text { ( } n=11) \\
\text { Indigestão: } 14,1 \% \text { ( } n=22) \\
\text { Mudança no padrão intestinal: } 19,9 \%(n=31) \\
\text { Redução na frequência das fezes: } 19,9 \% \\
\text { ( } n=31 \text { ) } \\
\text { Redução no volume das fezes: } 6,4 \%(n=10)\end{array}$ & $\begin{array}{l}\text { Hábitos de evacuação irregulares: 17,3\% ( } n=27) \\
\text { Ignoro habitualmente a urgência para evacuar: } \\
9 \%(n=14) \\
\text { Abuso de laxantes: } 1,3 \% \text { ( } n=2) \\
\text { Hábitos alimentares inadequados: } 31,4 \%(n=49) \\
\text { Ingestão insuficiente de fibras: } 25 \%(n=39) \\
\text { Ingestão insuficiente de líquidos: } 21,2 \%(n=33) \\
\text { Mudança nos hábitos alimentares ( } p . \text { ex., } \\
\text { alimentos, horário das refeições): } 17,9 \%(n=28) \\
\text { OUTROS MOTIVOS: } \\
\text { Intolerância alimentar, rotina acadêmica: } 3,2 \% \\
\text { (n=5) }\end{array}$ \\
\hline
\end{tabular}

FIGURA 1: Diagnósticos de Enfermagem mais prevalentes nos Estudantes de Graduação em Enfermagem. Rio Grande, RS, Brasil, 2018. 
Baseado nos diagnósticos de enfermagem mais prevalentes, traçaram-se os resultados a serem alcançados de acordo com a NOC e as intervenções necessárias para atingir tais resultados de acordo com a NIC, conforme apresentado na Figura 2.

\begin{tabular}{|c|c|c|}
\hline $\begin{array}{l}\text { Diagnósticos de } \\
\text { Enfermagem }\end{array}$ & Resultados Esperados (NOC) & Intervenções propostas (NIC) \\
\hline Fadiga & $\begin{array}{l}\text { Concentração } \\
\text { Conservação de Energia } \\
\text { Bem-Estar Pessoal } \\
\text { Estado de Autocuidados } \\
\text { Estado de Saúde Pessoal } \\
\text { Qualidade de Vida } \\
\text { Repouso } \\
\text { Sono }\end{array}$ & $\begin{array}{l}\text { Controle da Nutrição } \\
\text { Controle de Energia } \\
\text { Controle do Ambiente } \\
\text { Controle do Humor } \\
\text { Estabelecimento de Metas } \\
\text { Melhora do Sono } \\
\text { Promoção do Exercício } \\
\text { Melhora do Enfrentamento } \\
\text { Melhora do Sistema de Apoio } \\
\text { Redução da Ansiedade }\end{array}$ \\
\hline Insônia & $\begin{array}{l}\text { Bem-Estar Pessoal } \\
\text { Concentração } \\
\text { Equilíbrio do Humor } \\
\text { Estado de Saúde Pessoal } \\
\text { Qualidade de Vida } \\
\text { Repouso } \\
\text { Sono } \\
\text { Participação no Lazer } \\
\text { Estado de Conforto }\end{array}$ & $\begin{array}{l}\text { Controle da Nutrição } \\
\text { Facilitação da Meditação } \\
\text { Melhora do Enfrentamento } \\
\text { Musicoterapia } \\
\text { Promoção do Exercício } \\
\text { Redução da Ansiedade } \\
\text { Técnica para Acalmar } \\
\text { Treinamento de Autossugestão }\end{array}$ \\
\hline $\begin{array}{l}\text { Estilo de vida } \\
\text { sedentário }\end{array}$ & $\begin{array}{l}\text { Aptidão Física } \\
\text { Comportamento de Adesão } \\
\text { Comportamento de Promoção da Saúde } \\
\text { Conhecimento: Comportamento de Saúde } \\
\text { Conhecimento: Promoção da Saúde } \\
\text { Motivação } \\
\text { Participação no Lazer }\end{array}$ & $\begin{array}{l}\text { Facilitação da Autorresponsabilidade } \\
\text { Promoção de exercícios } \\
\text { Assistência na Automodificação }\end{array}$ \\
\hline Constipação & $\begin{array}{l}\text { Controle de Sintomas } \\
\text { Eliminação Intestinal } \\
\text { Função Gastrointestinal } \\
\text { Hidratação } \\
\text { Comportamento de Adesão: Dieta Saudável }\end{array}$ & $\begin{array}{l}\text { Explicar a etiologia do problema e a justificativa para as ações } \\
\text { Identificar os fatores (p. ex., medicamentos, repouso e dieta) que } \\
\text { possam causar ou contribuir para a constipação } \\
\text { Instituir um horário para o uso do vaso sanitário, conforme } \\
\text { apropriado } \\
\text { Encorajar o aumento da ingestão de líquidos, a menos que } \\
\text { contraindicado } \\
\text { Avaliar o perfil medicamentoso quanto a efeitos colaterais } \\
\text { gastrointestinais } \\
\text { Orientar o estudante/família sobre a relação entre dieta, } \\
\text { exercícios e ingestão de líquidos para constipação } \\
\text { Ensinar ao estudante/família os processos digestivos normais }\end{array}$ \\
\hline
\end{tabular}

FIGURA 2: Resultados esperados e Intervenções propostas para os Estudantes de Graduação em Enfermagem. Rio Grande, RS, Brasil, 2018.

\section{DISCUSSÃO}

Ao investigar as NHBs psicobiológicas em desequilíbrio nos estudantes, foi possível identificar que "sono e repouso" se encontram dentre as principais, visto que os dois diagnósticos de enfermagem mais prevalentes nos estudantes de enfermagem foram "Fadiga" e "Insônia". Ambos relacionados com estresse e ansiedade, que, segundo estudos científicos ${ }^{1,2,7}$, são características comumente encontradas em estudantes universitários, principalmente da área da saúde. Ainda, o estresse e a ansiedade são identificados como os principais responsáveis por insônia, que consequentemente geram fadiga, desmotivando os estudantes a realizarem as atividades acadêmicas ${ }^{1,2,7}$.

Estudantes da área da saúde possuem uma alta carga horária de atividades teóricas e práticas, portanto, têm menos tempo para desenvolver atividades de lazer ${ }^{23}$. Também são identificados como fatores geradores de estresse e ansiedade a indecisão frente à escolha do curso como profissão, despertando, assim, o sentimento de angústica, frustração, medo de não ser feliz e inclusive de não ter o retorno financeiro desejado ${ }^{24}$. 
Os participantes identificaram que os principais sinais apresentados por eles em relação ao diagnóstico de enfermagem de "fadiga" foram: cansaço; aumento da necessidade de descanso; e alteração na concentração. Já, relacionado à "insônia", os estudantes identificaram outros motivos além de estresse e ansiedade, como a rotina acadêmica e a jornada de trabalho noturna. Aspectos que afetam negativamente o desempenho acadêmico desses estudantes, gerando desmotivação perante o curso de Enfermagem. De acordo com um pesquisa, o rendimento acadêmico é drasticamente prejudicado pela falta de qualidade no sono, visto que repercute negativamente nas habilidades de concentração, memória e resolução de problemas ${ }^{25}$.

Outra consequência da baixa qualidade do sono é o aumento do apetite e, assim, o consumo de alimentos mais calóricos, ocasionando ganho de peso e possivelmente desenvolvimento de obesidade ${ }^{26}$. A obesidade está diretamente relacionada a um estilo de vida mais sedentário e ao aparecimento de disfunções gastrointestinais, tais como constipação ${ }^{27}$. Relação importante a ser observada, pois o número total de estudantes que estavam na classificação de sobrepeso e com obesidade nível I, II e III foi de 55, o que é preocupante.

Logo, percebe-se que o ingresso na universidade gera diversas mudanças na vida do estudante, tanto naqueles que somente estudam, exigindo mais autonomia e responsabilidade, quanto naqueles que possuem emprego, sendo necessária melhor organização do tempo ${ }^{1}$.

Para auxiliar os estudantes a enfrentarem de maneira positiva situações relacionadas ao desequilíbrio da NHB psicobiológica de "sono e repouso", a NIC dispõe de algumas possíveis intervenções a serem realizadas, como, por exemplo: facilitação da meditação, musicoterapia e técnica para acalmar ${ }^{11}$. Assim, cabe destacar que a NIC apresenta uma variedade das práticas de enfermagem e que algumas dessas irão requerer treinamento especializado ou certificação apropriada ${ }^{11}$, mas que se constituem importantes possibilidades de cuidado.

Em um estudo na Universidade Pública da Bahia, realizado com 353 estudantes do curso de graduação em enfermagem, encontrou-se que $85,0 \%$ desses foram considerados sedentários ${ }^{28}$. E em estudo na cidade de Porto, em Portugal, ao investigar a realização de atividade física por 535 estudantes de enfermagem, foi detectado que $27,6 \%$ dos participantes não possuíam prática esportiva ${ }^{29}$. De forma semelhante, em nosso estudo o "exercício e atividade física" encontravam-se em desequilíbrio nos estudantes, onde $42,7 \%$ dos participantes afirmaram não praticar atividade física. Logo, o terceiro diagnóstico de enfermagem mais prevalente identificado foi "Estilo de vida sedentário".

Dados preocupantes, visto que o sedentarismo é precursor de diversos problemas de saúde, dentre eles o principal é o desenvolvimento de doenças crônicas não transmissíveis, como dislipidemia, diabetes e hipertensão arterial. E se torna ainda mais perigoso quando associado a outros fatores de risco, como sobrepeso/obesidade ${ }^{30}$.

Outro diagnóstico de enfermagem prevalente nos estudantes refere-se à NHB psicobiológica "eliminação". Os principais fatores relacionados a este diagnóstico foram: ingestão insuficiente de fibras, líquidos e hábitos alimentares inadequados. Na literatura é possível encontrar resultados semelhantes, como é o caso de um estudo realizado em Curitiba, o qual investigou a incidência de constipação em estudantes universitários e verificou que 40 participantes, 47,5\%, apresentaram constipação, e relacionaram, principalmente, com a falta de tempo para manter hábitos alimentares adequados, observando-se uma correlação significativa entre constipação e ansiedade decorrente da rotina universitária ${ }^{31}$.

Dessa maneira, faz-se necessário o alerta para as instituições de ensino superior (IES), especialmente nos cursos de graduação em enfermagem, para identificarem os fatores que ocasionam o adoecimento dos estudantes, visto que diversos estão relacionados à rotina acadêmica. Bem como sugerir possíveis intervenções a serem realizadas, baseadas nas principais características apresentadas pelos estudantes ${ }^{31}$. Destaca-se, então, a importância da NIC neste estudo, visto que permite a delimitação de tópicos indispensáveis a serem abordados futuramente com estes estudantes, conforme apresentado na Figura 2.

\section{Limitações do estudo}

Como limitação do estudo, destaca-se que foi utilizada a amostra de conveniência, o que dificulta a generalização dos achados, representando apenas os estudantes de enfermagem da instituição onde foi realizada a pesquisa.

\section{CONCLUSÃO}

A partir deste estudo foi possível identificar que fadiga, insônia, estilo de vida sedentário e constipação foram os diagnósticos de enfermagem mais prevalentes nos participantes, na perspectiva das NHBs psicobiológicas. Além disso, foi possível descrever os resultados esperados e propor intervenções de enfermagem atingindo os objetivos propostos.

Observou-se que, dos quatro diagnósticos mais prevalentes, dois apresentavam o estresse e a ansiedade como fatores contribuintes para o surgimento, os quais merecem atenção para futuras intervenções. De acordo com a NIC algumas intervenções para esses fatores poderão ser: controle da nutrição, melhora do sono, promoção do exercício e facilitação da meditação. 
Torna-se importante o desenvolvimento de outros estudos na área, a fim de formar uma base científica ampla acerca do estado de saúde dos estudantes de enfermagem. E, para tal, a aplicação do Processo de Enfermagem e a utilização das taxonomias NANDA, NIC E NOC mostraram-se satisfatórias para identificar as NHBs em desequilíbrio e propor intervenções possíveis de serem realizadas nas IES. Almeja-se sensibilizar as IES em adotar medidas que promovam a saúde dos universitários, para formar profissionais capazes de priorizar o autocuidado.

\section{REFERÊNCIAS}

1. Moura IH, Nobre RS, Cortez RMA, Campelo V, de Macêdo SF, da Silva ARV. Quality of life of undergraduate nursing students. Rev. gaúch. enferm. 2016 [cited 2020 Feb 17]; 37(2):255-91. DOI: http://dx.doi.org/10.1590/1983-1447.2016.02.55291.

2. The WHOQOL Group. The World Health Organization quality of life assessment (WHOQOL): position paper from the World Health Organization. Soc. sci. med. 1995 [cited 2020 Feb 17]; 41:1403-10. Available from: https://pubmed.ncbi.nlm.nih.gov/8560308/.

3. Gomes KK, Sanchez HM, Sanchez EGM, Sbroggio Júnior AL, Arantes Filho WM, Silva LA. et al. Quality of life and quality of working life of health science professors at a higher education institution. Rev. bras. med. trab. 2017 [cited 2020 Apr 23]; 15(1):18-28. Available from: http://docs.bvsalud.org/biblioref/2019/04/833205/v15n1a04_0kg2Zoi.pdf.

4. Chazan ACS, Campos MR, Portugal FB. Quality of life of medical students at the State University of Rio de Janeiro (UERJ), measured using Whoqol-bref: a multivariate analysis. Cienc. Saude Colet. 2015 [cited 2020 Feb 17]; 20(2):547-56. DOI: https://doi.org/10.1590/1413-81232015202.05182014.

5. Cunha M, Duarte J, Sandré S, Sequeira C, Castro Molina FJ, Mota M. et al. Well-being in high school students. Millenium. 2017 [cited 2020 Apr 23]; 2(esp.2):21-38. DOI: https://doi.org/10.29352/mill0202e.02.

6. Hirsch CD, Barlem ELD, de Almeida LK, Tomaschewski-Barlem JG, Lunardi VL, Ramos AM. Stress triggers in the educational environment from the perspective of nursing students. Texto \& contexto enferm. 2018 [cited 2020 Apr 14]; 27(1):1-11. DOI: http://dx.doi.org/10.1590/0104-07072018000370014.

7. Moritz AR, Pereira EM, Borba KP, Clapis MJ, Gevert VG, Mantovani MF. Quality of life of undergraduate nursing students at a Brazilian public university. Invest. educ. enferm. 2016 [cited 2020 Apr 23]; 34(3):564-72. DOI: https://doi.org/10.17533/udea.iee.v34n3a16

8. Hart PL, Brannan JD, de Chesnay M. Resilience in nurses: an integrative review. J. nurs. manag. 2014 [cited 2020 Aug 28]; 22:720-34. DOI: https://doi.org/10.1111/j.1365-2834.2012.01485.x.

9. Reeve KL, Shumaker CJ, Yearwood EL, Crowell NA, Riley JB. Perceived stress and social support in undergraduate nursing students' educational experiences. Nurse educ. today. 2013 [cited 2020 Aug 28]; 33:419-24. DOI: https://doi.org/10.1016/j.nedt.2012.11.009.

10. Thomas L, Revell SH. Resilience in nursing students: an integrative review. Nurse educ. today. 2016 [cited 2020 Aug 28]; 36:457-62. DOI: https://doi.org/10.1016/j.nedt.2015.10.016.

11. Khamisa N, Oldenburg B, Peltzer K, llic D. Work related stress, burnout, job satisfaction and general health of nurses. Int. j. environ. res. public health. 2015 [cited 2020 Aug 28]; 12:652-66. DOI: https://doi.org/10.1111/ijn.12455.

12. Silva EC, Heleno MGV. Quality of Life and Subjective Well-Being of College Students. Rev. psicol. saúde. 2012 [cited 2020 Feb 02]; 4(1):69-76. Available from: https://www.unifesp.br/reitoria/prae/publicacoes/publi/producao-cientifica-assistenciaestudantil/artigos?download=255:qualidade-vida.

13. World Health Organization. WHOQOL: measuring quality of life. The WHOQOL Group. Geneva; 1997 [cited 2020 Abr 23]. Available from: http://www.who.int/mental_health/media/68.pdf

14. Fleck MPA, Louzada S, Xavier M, Chachamovich E, Vieira G, Santos L. et al. Aplicação da versão em português do instrumento abreviado de avaliação da qualidade de vida WHOQOL-bref. Rev. Saúde Pública [Internet], 2000 [cited 2020 feb 02]; 34(2):17883. Available from: http://www.scielo.br/scielo.php?script=sci_arttext\&pid=S0034-89102000000200012.

15. Conselho Nacional de Saúde (Br). Resolução no 466 de 12 de dezembro de 2012. Diretrizes e normas regulamentadoras de pesquisas envolvendo seres humanos. Brasília (DF): CNS; 2012. [cited 2020 Feb 02]. Available from: http://conselho.saude.gov.br/resolucoes/2012/Reso466.pdf.

16. Silva EC, Tucci AM. Brief intervention to reduce alcohol consumption and its consequences in Brazilian university students. Psicol. reflex. crit. 2015 [cited 2020 Apr 23]; 28(4):728-36. DOI: https://doi.org/10.1590/1678-7153.201528410.

17. Pires CG, Mussi FC, Souza RC, Silva DO, Santos CA. Consumption of alcohol among nursing students. Acta paul. enferm. 2015 [cited 2020 Apr 23]; 28(4):301-7. DOI: http://dx.doi.org/10.1590/1982-0194201500052.

18. Costa DD, Ferreira NIB. PROUNI (University for All Program) in Brazilian Higher Education: indicators of access and permanence. Avaliação (Campinas; Sorocaba). 2017 [cited 2020 Oct 10]; 22(1):141-63. DOI: http://dx.doi.org/10.1590/S141440772017000100008

19. Sawicki WC, Barbosa DA, Fram DS, Belasco AGS. Alcohol consumption, quality of life and brief intervention among nursing university students. Rev. bras. enferm. 2018 [cited 2020 Apr 21]; 71(supl.1):547-55. DOI: http://dx.doi.org/10.1590/0034-71672017-0692.

20. Lubman DI, Garfield JB, Manning V, Berends L, Best D, Mugavin JM. et al. Characteristics of individuals presenting to treatment for primary alcohol problems versus other drug problems in the Australian patient pathways study. BMC psychiatry. 2016 [cited 2020 Apr 23]; 16:250. DOI: https://doi.org/10.1186/s12888-016-0956-9.

21. Damasceno RO, Boery RN, Ribeiro IJ, Anjos KF, Santos VC, Boery EN. Use of alcohol, tobacco and other drugs, and quality of life among college students. Rev. baiana enferm. 2016 [cited 2017 May 20]; 30(3):1-10. DOI: 
https://doi.org/10.18471/rbe.v30i3.15533.

22. Costa DG, Carleto CT, Santos VS, Haas VJ, Gonçalves RMDA, Pedrosa LAK. Quality of life and eating attitudes of health care students. Rev. Bras. Enferm. 2018 [cited 2020 Aug 28]; 71(supl.4):1739-46. DOI: https://doi.org/10.1590/0034-7167-2017-0224.

23. Gama ASM. Quality of life of nursing students from Amazonas, Brazil. Rev. baiana enferm. 2016 [cited 2020 Apr 23]; 30(4):1-9. DOI: https://doi.org/10.18471/rbe.v30i4.17011.

24. Freitas AMC, Bárbara JFRS, do Vale PRLF, Carvalho SS, de Carvalho LF, Nery GS. Undergraduate nursing students perceptions in relation to quality of life in academic trajectory. Rev. enferm. UFSM. 2017 [cited 2020 Apr 23]; 7(2):152-66. DOI: https://doi.org/10.5902/2179769225391.

25. Peltzer K, Pengpid S1. Sleep duration and health correlates among university students in 26 countries. Psychol. health med. 2016 [cited 2020 Aug 28]; 21(2):208-20. DOI: https://doi.org/10.1080/13548506.2014.998687.

26. Chow KM, Tang WKF, Chan WHC, Sit WHJ, Choi KC, Chan S. Resilience and well-being of university nursing students in Hong Kong: a cross-sectional study. BMC medical educ. 2018 [cited 2020 Aug 29]; 18(13):01-08. https://bmcmededuc.biomedcentral.com/articles/10.1186/s12909-018-1119-0.

27. Simões AV, Penna LHG, Rodrigues VP, Carinhanha JI, Pereira ALF, Machado JC. Young university students' intimate affective relationships: nursing students' narratives. Rev. enferm. UERJ. 2019 [cited 2020 Apr 23]; 21:1-06. DOI: https://doi.org/10.12957/reuerj.2019.34355.

28. Pavão AL, Werneck GL, Campos MR. Self-rated health and the association with social and demographic factors, health behavior, and morbidity: a national health survey]. Cad. saúde pública. 2013 [cited 2020 Apr 23]; 29(4):723-34. Available from: http://www.scielosp.org/pdf/csp/v29n4/10.pdf.

29. Bublitz S, Guido LA, Lopes LFD, Freitas EO. Association between nursing students academic and sociodemographic characteristics and stress. Texto \& contexto enferm. 2016 [cited 2020 Apr 23]; 25(4):1-7. DOI: http://dx.doi.org/10.1590/010407072016002440015. 